Elsevier required licence: (C) $<2020>$. This manuscript version is made available under the CC-BY-NCND 4.0 license http://creativecommons.org/licenses/by-nc-nd/4.0/

The definitive publisher version is available online at

[https://www.sciencedirect.com/science/article/pii/S0965229920305112?via\%3Dihub] 


\title{
Perceptions of Person-Centred Care Amongst Individuals with Chronic Conditions who Consult Complementary Medicine Practitioners
}

\begin{abstract}
Objectives: Chronic conditions require continuous, multi-factorial care - such as personcentred care - to address patients' individual health needs and quality of life. Many patients with chronic conditions seek additional care outside mainstream medicine, often consulting complementary medicine $(\mathrm{CM})$ practitioners. This study examines person-centred care experienced by patients with chronic conditions consulting CM practitioners. Design: Crosssectional survey. Setting: CM clinics around Australia, conducted November 2018 to March 2019. Participants: Patients with chronic conditions $(n=153)$ consulting osteopaths $(n=39)$, naturopaths $(n=33)$, massage therapists $(n=29)$, chiropractors $(n=28)$ and acupuncturists ( $\mathrm{n}=24)$. Main Outcome Measures: Patient-Centred Care Scale, Perceived Provider Support Scale, Empowerment Scale, and Patient Assessment of Chronic Illness Care measure. Results: Patient perceptions of person-centred care were consistently high during consultation with CM practitioners (Patient-centred Care scale mean range 4.22 to 4.70; Perceived Provider Support scale mean range 4.39 to 4.69; Empowerment scale mean range 2.20 to 2.50; Patient Assessment of Chronic Illness Care mean summary 3.33). Ratings of personcentred care were higher for consultations with CM practitioners than for medical doctors. Patients of naturopaths reported the highest means for perceived person-centred care. Variation in participant ratings for different items between professions indicate nuance in
\end{abstract}


consultation experiences across different CM professions. Conclusions: Person-centred care appears characteristic of $\mathrm{CM}$ consultation, which may reflect holistic philosophies. Variations in patient experiences suggest diverse practices across CM professions. CM practitioners may present a resource of person-centred care for addressing unmet needs of individuals with chronic conditions, and reducing the health burden associated with rising rates of chronic conditions.

Keywords: patient-centred care, chronic illness, complementary medicine, patient experience, public health, clinical care. 


\section{Introduction}

Rates of chronic condition diagnoses have risen in recent years and increasingly contribute substantial burden to health care systems globally. ${ }^{1}$ Chronic conditions are prolonged in duration, typically complex in causes and symptoms, and impact on patients' lives in a variety of ways. ${ }^{2}$ As well as requiring direct medical intervention, many chronic conditions leave patients with reduced functional, social and economic capacity which can further impact families and communities. ${ }^{3}$ Optimal chronic disease management requires continuous, individualised and multi-faceted approaches to clinical care beyond treatment of pathology in order to address the prolonged, complex nature of chronic conditions and to account for the pervasive effects on patients' quality of life. ${ }^{3}$ In particular, comprehensive, individualised consultation approaches ${ }^{4}$ and interventions designed to strengthen patientprovider relationships ${ }^{5}$ show favourable effects on clinical outcomes in chronic conditions.

Due to the historical and contemporary primary focus of health care systems upon treating acute and infectious diseases - previously presenting the greatest contribution to the burden of disease ${ }^{1}$ - there are numerous challenges to adjusting to better manage chronic conditions. ${ }^{6,7}$ Person-centred care (PCC) has been recognised in a number of public health policies and guidelines as a paradigm of clinical care with potential to provide the necessary adjustments to care provision for this purpose. ${ }^{3,8}$ PCC seeks to account for the multifactorial aspects of health management by delivering clinical care that is "respectful of, and responsive to, individual patient preferences, needs and values, and ensuring that patient values guide all clinical decisions". ${ }^{9}$ This respect and responsiveness to individual patient circumstances in PCC attends to the complexity of chronic conditions ${ }^{3}$ and could facilitate development of the efficiency and sustainability required for health systems to adequately manage increasing chronic illness. ${ }^{8}$ 
An unmet desire for respectful, responsive, individualised clinical care can lead patients with chronic conditions to seek such care outside of mainstream health care systems, often via consultation with complementary medicine $(\mathrm{CM})$ practitioners. ${ }^{10}$ Those with chronic conditions may seek the care of CM practitioners specifically to address side-effects from, or dissatisfaction with, conventional treatment, as well as for management of their condition from an holistic perspective. ${ }^{11}$-Many CM professions adhere to practices founded upon the philosophy of holism, which seeks to treat the "whole person" rather than simply addressing a patient's pathology. ${ }^{12}$ Holism is well-aligned with PCC due to this whole-person approach $^{12}$ which may lead to PCC being consequently delivered in CM clinical practice. As patients with chronic conditions tend to demonstrate higher utilisation of CM practitioner services than the general population, ${ }^{11}$ it is imperative to determine whether $\mathrm{CM}$ clinical practice translates to experiences of PCC for patients.

\section{Methods}

The present study sought to determine the extent to which patients with chronic conditions experience PCC during consultation with CM practitioners.

\subsection{Study Design and Setting}

A cross-sectional survey was conducted between November 2018 and March 2019, in CM clinics throughout Australia. The CM professions chosen for the study were identified through previous research ${ }^{13}$ as the five most frequently consulted clinical CM professions in Australia and included chiropractic, massage therapy, osteopathy, acupuncture and naturopathy. Invitations to assist with study patient recruitment were sent to practitioners of these professions through three practitioner-based research networks (PBRNs): the Practitioner Research and Collaboration Initiative (PRACI), ${ }^{14}$ the Osteopathy Research and 
Innovation Network (ORION), ${ }^{15}$ and the Australian Chiropractic Research Network $(\mathrm{ACORN}) .{ }^{16}$

\subsection{Participants and Recruitment}

Expressions of interest and consent forms were submitted online by CM practitioners who were members of the participating PBRNs and were in active clinical practice. From each of the five professions, seven to eight practitioners were selected based on location to achieve a broad geographical spread. Selected practitioners were provided with hardcopy study materials which included information sheets, surveys to distribute, and instructions detailing the study protocol, recruitment process and scripted guidelines for communicating with patients about the study. Each practitioner then distributed a study information sheet, consent form and hardcopy survey to 15 consecutive eligible patients, who were invited to self-administer the survey at a time and place convenient to them after leaving the clinic. This approach allowed patients to participate (or not) anonymously (blinding practitioners to recruitment outcomes) and without practitioner coercion to reduce selection bias. This recruitment process was chosen to ensure the integrity of patient-practitioner relationships was not affected by the study.

Eligibility criteria for patient participation required that patients be adults (aged 18 and over), fluent in the English language, capable of providing consent, and had not already completed the survey during a previous consultation with the CM practitioner. The surveys included a reply-paid postage envelope for return directly to the research team at no cost to patients or recruiting practitioners. Each survey included a web-link where participating patients could enter a draw to win a $\$ 100$ gift-voucher as an incentive to participate. Sample size calculations were undertaken based on response rates from previous research ${ }^{17}$ conducted with a similar population and conservative estimates of chronic condition 
prevalence, resulting in a desired sample of 377 patients being sought to achieve a $5.0 \%$ margin of error.

\subsection{Instrument}

The survey included 29 questions in total, covering socio-demographics, current chronic condition diagnoses, details of $\mathrm{CM}$ practitioner service utilisation, patient experiences of care in CM and conventional medical settings with medical doctors (MDs - general practitioners or specialist doctors), and patient-practitioner communication about patient treatment and medication use. Respondents who did not report a current chronic condition diagnosis were only asked to complete socio-demographic items. Items applicable to the analyses presented here included socio-demographics, chronic condition diagnoses and patient experiences of care.

Socio-demographics covered age, gender, state of residence, relationship status, educational qualification level, employment status, financial manageability, private health insurance (PHI) coverage, and possession of a Health Care Card (provided to low-income earners and welfare recipients in Australia for health and medical financial concessions). Diagnoses of chronic conditions were identified by respondents from a list (arthritis, asthma, cancer or post-cancer treatment complications, chronic obstructive pulmonary disease, diabetes type 1 , diabetes type 2 , heart disease/cardiovascular disease, hypertension/high blood pressure, musculoskeletal condition, chronic pain, depression, anxiety, other mental health condition, insomnia or other sleep disorder, obesity, Alzheimer's disease or dementia, congenital condition, chronic kidney disease, endometriosis, polycystic ovarian syndrome, other female reproductive disorder, inflammatory bowel disease, irritable bowel syndrome, fibromyalgia of chronic fatigue syndrome/myalgic encephalomyelitis, other autoimmune disease), with additional options for open-text responses. 
Measures pertaining to patient experiences of care included the Patient-Centred Care Scale (PCCS), Perceived Provider Support Scale (PPSS), Empowerment Scale and the Patient Assessment of Chronic Illness Care (PACIC) measure. The PCCS, PPSS and Empowerment Scale are designed to be co-administered interdependently. ${ }^{18}$ The PCCS (ten items) and PPSS (seven items) allow patients to rate aspects of person-centred care and perceived emotional support from the provider (respectively) across a five-point Likert scale from Strongly disagree (value of 1) to Strongly agree (value of 5), while the Empowerment Scale (five items) allows patients to rate the sense of health-related empowerment resulting from the consultation across a three-point scale of No (value of 1), Yes a little (value of 2), and Yes a lot (value of 3). ${ }^{18}$ The PACIC measure includes twenty items using a five-point scale ranging from Almost never (value of 1) to Almost always (value of 5) which allow patients to rate five domains of actions and clinical care qualities of person-centred care as they relate specifically to management of chronic conditions (patient activation, delivery system design/decision support, goal setting/tailoring, contextual problem-solving, followup/coordination). ${ }^{19}$ The survey asked patients to complete these four measures in response to the consultation they had just attended with their CM practitioner, as well as in relation to their most recent consultation with their MD (general practitioner or specialist doctor, if applicable).

\subsection{Data Handling and Analysis}

Analyses were completed using StataIC 14 (StataCorp LC 2015). Analyses presented here utilised data from respondents who reported one or more chronic condition diagnoses. Some socio-demographic variables were collapsed and recoded to produce adequate cell sizes, when appropriate (age, relationship status, educational qualification, employment status, financial manageability). CM professions consulted were recoded as binaries to allow 
comparisons between characteristics of participants who had consulted with different professions using Fisher's exact test.

Descriptive statistics were tabulated as frequencies and percentages, while summary statistics were calculated as means and standard deviations. Summary statistics were calculated for each item in the PCCS, PPSS and Empowerment Scale with missing responses excluded from analysis for that item. The PACIC measure was scored by calculating means for each item and each domain, as well as across the full measure to provide a summary score, in accordance with the measure's intended use and previous validation. ${ }^{19}$ During calculation of PACIC domain scores and summary scores, observations with more than one missing value per domain were excluded; these observations were included in single item calculations.

\subsection{Ethics}

Ethical approval was granted by the Human Research Ethics Committee, University of Technology Sydney (ETH18-2769).

\section{Results}

Thirty-nine CM practitioners (seven chiropractors and eight practitioners from each other profession) assisted with recruitment of patients by distributing surveys and confirming recruitment completion. In total, 585 patient surveys were distributed and 199 were returned to the research team (response rate $34.0 \%$ ). Of the returned surveys, eight were excluded due to an excess of missing responses or contradictory responses which threatened data reliability. Of the remaining 191 surveys, 38 reported no chronic condition diagnoses and were excluded from the present analyses, producing a final sample of 153.

\subsection{Participant Characteristics}


Participants with chronic conditions were most commonly female $(n=126,82.4 \%)$, aged 65 years and over $(n=44,29.0 \%)$, married $(n=85,55.9 \%)$, vocationally or trade qualified $(n=61,40.1 \%)$, not in the paid workforce $(n=57,37.5 \%)$ and reported financial manageability to be Not too bad ( $\mathrm{n}=72,48.0 \%$ ). Participants predominantly held PHI cover generally $(n=120,79.0 \%)$ and specifically for the CM profession they were consulting $(n=108,71.1 \%)$, while just over one-third held Health Care Cards $(n=56,37.6 \%)$.

Comparisons between those consulting practitioners from different professions found a higher proportion of men consulting chiropractors $(p=0.024)$ and a higher proportion of participants from the 65 years and over age group consulting naturopaths $(p=0.023)$. Those consulting naturopaths also had a significantly lower rate of PHI coverage both generally $(p<0.001)$ and for the CM profession specifically $(p=0.001)$. Those consulting massage therapists had lower rates of Health Care Card coverage $(p=0.027)$. See Table 1 .

\subsection{Patient-centred Care}

Perceptions of PCC during consultation with CM practitioners were consistently high across the PCCS. For the total sample (consulting any CM practitioner), the highest mean score (of a possible 5.00) was for the item My practitioner is really interested in finding and addressing my health problems (mean 4.70), and the lowest was for The root causes of my problems are being treated by my practitioner (mean 4.22) (full details in Table 2). Those within the total sample who had consulted a MD reported lower perceptions of PCC for MD consultations, with the highest mean at 4.18 for item My doctor is really interested in finding and addressing my health problems, ranging to the lowest mean of 3.45 for item My doctor teaches me ways to relieve symptoms myself.

Table 2 also reports the profession-specific results for whole-system CM professions acupuncture and naturopathy. Patients consulting acupuncturists reported higher means for 
items such as I know what to expect during treatment sessions and lower means for items such as My practitioner teaches me ways to relieve my symptoms myself. Those consulting naturopaths reported a higher mean for the item My practitioner teaches me ways to relieve my symptoms myself and a lower mean for My practitioner receives feedback from my body that guides treatment. When compared to the total sample, patients of naturopaths reported lower means for their MD consultations across all items of the PCCS.

Table 3 reports the profession-specific results for manual therapy CM professions - massage therapy, osteopathy and chiropractic. Patients of massage therapists reported higher means for MD consultations across all items and patients of osteopaths reported higher means for eight out of ten items. Patients of chiropractors and osteopaths reported higher means for item The root causes of my problems are identified by my practitioner, while patients of massage therapists and osteopaths reported higher means for My practitioner receives feedback from my body that guides treatment.

\subsection{Perceived Provider Support}

For the total sample consulting any CM practitioner, perceptions of provider support were strong, with the highest mean (of a possible 5.00) recorded for the item I trust my practitioner (mean 4.69) and the lowest mean for My practitioner gives me hope (mean 4.39) (full details in Table 2). Strongly agree/agree responses to the PPSS items were highly consistent, recorded by a majority of respondents across all items. No respondents selected Strongly disagree/disagree for any PPSS items regarding consultations with their CM practitioner (see Supplementary Table S1).

In comparison to $\mathrm{CM}$ consultations, respondents who had consulted a MD reported lower means for their medical consultation across all PPSS items. The items attracting the 
highest and lowest means were I trust my doctor (4.32) and My doctor gives me hope (3.77) respectively (see Table 2).

Compared to the total sample, participants consulting acupuncturists and massage therapists reported higher means across all items of the PPSS for their CM consultation, while all means for those consulting chiropractors were slightly lower. Naturopathy patients reported lower means for all items in response to their consultations with MDs (Table 2), while patients of massage therapists and osteopaths reported higher means for their MDs for all items in the PPSS (Table 3), compared with the total sample.

\subsection{Patient Empowerment}

Responses to the Empowerment Scale for respondents consulting any CM practitioner were typically favourable, with the highest mean (of a possible 3.00) reported for item $D o$ you know what to do to take care of your health problem (mean 2.50) and the lowest mean reported for Do you advocate more for yourself (mean 2.20) (Table 2). The categorical presentation of these items are reported in Supplementary Table S1.

For participants who responded to the Empowerment Scale regarding consultations with MDs, means were lower than those for CM consultations for all items. The item Do you know what to do to take care of your health problem achieved the highest mean (2.35), while item Do you believe your health problem will improve achieved the lowest mean (1.88) (Table 2).

Higher means were reported for all five items by patients consulting naturopaths (Table 2) and for four items by those consulting osteopaths (Table 3), compared to the total sample for $\mathrm{CM}$ consultations. Regarding MD consultations, higher means were reported by those consulting acupuncturists (Table 2), massage therapists and osteopaths, while lower 
means were reported by those consulting chiropractors (Table 3), compared to the total sample.

\subsection{Patient Assessment of Chronic Illness Care}

The total summary score mean for the PACIC measure, across the total sample, was 3.33 for consultations with any CM practitioner, and 2.95 for consultations with a MD. The highest summary score mean was reported for consultations with naturopaths (4.04) while the lowest was reported by patients of naturopaths for consultations with their MDs (2.84). Of the five PACIC domains, the domain attracting the highest summary score for consultations with CM practitioners was Delivery and practice (mean 3.87), while the domain attracting the highest summary score for consultations with MDs was Patient activation (mean 3.38). The domain Follow up and coordination attracted the lowest summary score for both CM consultations (mean 2.66) and conventional medicine consultations (mean 2.52) (Table 4). A general trend demonstrated higher summary scores for consultations with CM practitioners compared to consultations with MDs, with the exception of responses from patients of massage therapists, who reported slightly higher total scores for their MD (mean 3.27) compared to their massage therapist (mean 3.23) (Table 5).

Domain summary score means were highest for consultations with naturopaths across all five domains, ranging from 3.34 for Follow up and coordination to 4.50 for Delivery and practice (Table 4). The lowest summary scores for domains Patient activation (mean 3.02) and Delivery and practice (mean 3.13) were both recorded for MD consultations for chiropractic patients (Table 5). The lowest summary scores for domains Goal setting and tailoring (mean 2.66) and Problem-solving and contextual counselling (mean 2.93) were both recorded for MD consultations for naturopathy patients (Table 4), while the lowest summary 
score for domain Follow up and coordination (mean 2.34) was recorded for chiropractic consultations (Table 5).

\section{Discussion}

This study is the first to provide detailed reporting of experiences of PCC specific to individuals with chronic conditions who consult with CM practitioners, across a variety of clinical professional settings. Many participants reported experiencing PCC during consultation with CM practitioners. While there was some nuanced variation between groups consulting with different $\mathrm{CM}$ professions, each of the five professions attracted consistently high ratings of PCC overall from patients for all four measures. This is reflective of existing literature suggesting that PCC is generally characteristic of CM consultations. ${ }^{17,20}$

Across all measures assessing patient perceptions of the care they received, respondents also consistently reported higher ratings for their experience of consultations with CM practitioners compared to consultations with MDs, which may be influenced by different practical approaches within conventional and complementary medicine systems.

Due to its development being centred on addressing the historical burdens of acute and infectious diseases, the conventional medical system faces many challenges in moving toward a model of care provision that adequately addresses the needs of those with chronic conditions. ${ }^{1}$ In contrast, it has been suggested that CM philosophies have contributed to the development of $\mathrm{PCC},{ }^{21}$ which as a paradigm of clinical care is particularly well-suited to chronic illness management. ${ }^{22}$ Indeed, the philosophy of holism which underlies many CM professions appears to correlate closely in principle with PCC. ${ }^{12} \mathrm{CM}$ practitioners also tend to provide longer consultations than $\mathrm{MDs},{ }^{20}$ allowing more time for patients and practitioners to explore the complex, multifactorial needs of individuals with chronic conditions. Previous studies have identified the lengthier consultations of CM services such as naturopathy and 
acupuncture as contributing to patients feeling heard, and to patients perceiving $\mathrm{CM}$ practitioners as caring and trustworthy, ${ }^{23,24}$ which is congruous with the perceptions of CM practitioners reported by our participants. Thus, it may be that applied holistic philosophy and lengthier consultation time both contributed to our participants' reporting higher PCC in CM consultations compared to those with MDs. Clinical settings which provide holistic CM care alongside conventional medicine have demonstrated that such integration of services may be an avenue through which to enhance patient-centredness, as well as both patient and provider satisfaction. $^{25-27}$

For the majority of items across the survey, care received from naturopaths was rated most highly by respondents. While there are any number of factors that may be contributing to this finding, a previous review of patient perceptions of care in CM proposed the importance of patient empowerment and facilitation of patient self-efficacy in some CM professions may be key. ${ }^{17}$ The other four CM professions consulted by our participants employ primarily practitioner-enacted treatments during consultation (e.g. direct application of manual therapies or acupuncture needles), demonstrated in the lower mean reported by acupuncture patients for the item My practitioner teaches me ways to relieve symptoms myself and higher means reported by patients of massage therapists and osteopaths for the item My practitioner receives feedback from my body that guides treatment. Naturopaths, however, rely largely on patient-enacted treatments (e.g. remedies or dietary/lifestyle advice that patients must self-administer outside of the consultation) - an approach requiring the naturopath to engage the patient in the treatment process, which typically involves provision of patient education and detailed discussion of the patient's individual circumstances. ${ }^{28}$ Previous studies show such patient education by naturopaths may improve the patient's selfefficacy and sense of empowerment, while time spent discussing the patient's needs may 
result in the patient feeling heard and supported, ${ }^{17,23}$ leading to a particularly high degree of perceived PCC during consultation with naturopaths.

While care provided by $\mathrm{CM}$ practitioners was typically rated more highly than care provided by MDs, patients of different CM professions differed slightly in the domains and items for which they gave lower ratings to their MDs. For example, across the PACIC measure, compared to patients of other CM professions, patients of naturopaths rated their MDs lower in the domain of Problem solving and contextual counselling and patients of chiropractors rated their MDs lower in Patient activation. This may speak to differences in patients' unmet needs potentially prompting patients to seek care from particular CM professions - with those professions possibly being perceived as more likely to meet a specific unmet need. The individualised approach of PCC, however, is intended to facilitate the meeting of individual needs regardless of the care provider's profession. ${ }^{29}$

\subsection{Implications}

Our finding that $\mathrm{CM}$ practitioner consultations were characteristically person-centred for our participants correlates with the existing body of literature identifying aspects of CM consultation which are aligned with PCC. ${ }^{20}$ Patients with chronic conditions have expressed a desire and need for more person-centred approaches to their care, ${ }^{30}$ while person-centred aspects of clinical care such as provider empathy, strong communication ${ }^{5}$ and personalised consultations/treatments ${ }^{4}$ have been correlated with favourable health and psychosocial outcomes. The utility and importance of PCC in management of chronic conditions has been recognised in international ${ }^{8}$ and national ${ }^{3,9}$ health policy and guidelines, due to its capacity to address complex presentations or underlying aspects of illness such as those seen in chronic conditions. As chronic illness increasingly contributes to the burden of disease, and as patients with chronic conditions continue to seek multiple sources of care to manage their 
complex needs, it should be considered that $\mathrm{CM}$ practitioners may represent an existing resource of person-centred clinical management to address otherwise unmet aspects of care for this patient population.

\subsection{Limitations}

The results of this study provide promising insights into the potential benefits of CM consultations for individuals with chronic conditions, yet certain limitations must be noted. The small sample size and convenience sampling method preclude the use of more robust statistical analyses, while a suboptimal response rate potentially indicates presence of nonresponse bias, limiting the capacity for generalisation of findings. However, the broad geographical spread of clinic locations enhances representativeness, while the consecutive approach to recruitment moderates risk of sampling bias.

Due to small numbers in sub-groups delineated by CM profession consulted, alongside dependency of sub-groups separated by CM vs. MD consults (i.e. patients consulting MDs were the same patients consulting CM practitioners), statistical tests of association or comparison regarding the four measures used were not possible. Future research examining such comparisons should be conducted using larger, independent samples. Additionally, participant responses rating consultations with CM practitioners and with MDs may be impacted by recall bias as the survey was administered directly following $\mathrm{CM}$ consultation and the time period between survey and consultations with MDs will have been more extensive (in some cases perhaps sizeable). While self-report survey research always carries a risk of response and non-response bias, the consistency of results demonstrated by this study provide compelling rationale for further attention to and research in this area.

\section{Conclusion}


Our findings demonstrate notably favourable and consistent patient perceptions of PCC in CM clinical settings for individuals with chronic conditions. It appears the patient experience of PCC is characteristic of CM clinical care to a greater extent than in conventional medical settings. In light of the challenges presented to health systems by the rising rates, complexity and ongoing nature of chronic conditions, consideration should be given to the value CM professionals may contribute to addressing such challenges by providing individualised, tailored care to their patients.

\section{Acknowledgements}

The authors wish to thank the administration and practitioner members of the practitionerbased research networks - PRACI, ORION and ACORN - who assisted with recruitment for this project, as well as survey participants. The time given voluntarily to this work is deeply appreciated.

\section{Funding}

This work was supported by the Faculty of Health, University of Technology Sydney, through the Health Services and Practice Research Student Development Award (funding provided to H.F.). While working on this project, HF was supported by an Australian Government Research Training Program Scholarship and JA was supported by an Australian Research Council Professorial Future Fellowship (Grant FT140100195). Funding sources had no input into the design, conduct or reporting of this study, or in the decision to submit this article for publication.

\section{Declaration of interests}

None. 


\section{References}

1. World Health Organization. Global status report on noncommunicable diseases 2010.

Geneva: World Health Organization; 2011.

2. Goodman RA, Posner SF, Huang ES, Parekh AK, Koh HK. Defining and measuring chronic conditions: imperatives for research, policy, program, and practice. Prev Chronic Dis. 2013;10: E66.

3. Australian Health Ministers' Advisory Council. National strategic framework for chronic conditions. In: Department of Health, ed. Canberra: Australian Government; 2017.

4. Dossett ML, Mu L, Davis RB, et al. Patient-provider interactions affect symptoms in gastroesophageal reflux disease: a pilot randomized, double-blind, placebo-controlled trial. PLoS One. 2015;10(9): e0136855.

5. Kelley JM, Kraft-Todd G, Schapira L, Kossowsky J, Riess H. The influence of the patientclinician relationship on healthcare outcomes: a systematic review and meta-analysis of randomized controlled trials. PLoS One. 2014;9(4): e94207.

6. Harris MF, Zwar NA. Care of patients with chronic disease: the challenge for general practice. Med J Aust. 2007;187(2): 104-107.

7. Bodenheimer T, Chen E, Bennett HD. Confronting the growing burden of chronic disease: can the US health care workforce do the job? Health Aff. 2009;28(1): 64-74.

8. World Health Organization. WHO global strategy on people-centred and integrated health services: Interim report. Geneva: World Health Organization; 2015.

9. Committee on Quality of Health Care in America. Crossing the quality chasm: A new health system for the 21st century: National Academies Press; 2001.

10. Mak JCS, Faux S. Complementary and alternative medicine use by osteoporotic patients in Australia (CAMEO-A): a prospective study. J Altern Complement Med. 2010;16(5): 579-584.

11. Reid R, Steel A, Wardle J, Trubody A, Adams J. Complementary medicine use by the Australian population: a critical mixed studies systematic review of utilisation, perceptions and factors associated with use. BMC Complement Altern Med. 2016;16(1): 176. 
12. Foley $\mathrm{H}$, Steel $\mathrm{A}$. The nexus between patient-centered care and complementary medicine: Allies in the era of chronic disease? J Altern Complement Med. 2017;23(3): 158-163.

13. Steel A, McIntyre E, Harnett J, et al. Complementary medicine use in the Australian population: Results of a nationally-representative cross-sectional survey. Sci Rep. 2018;8(1): 17325 . 14. Steel A, Sibbritt D, Schloss J, et al. An overview of the Practitioner Research and Collaboration Initiative (PRACI): a practice-based research network for complementary medicine. BMC Complement Altern Med. 2017;17(1): 87.

15. Adams J, Sibbritt D, Steel A, Peng W. A workforce survey of Australian osteopathy: analysis of a nationally-representative sample of osteopaths from the Osteopathy Research and Innovation Network (ORION) project. BMC Health Serv Res. 2018;18(1): 352.

16. Adams J, Peng W, Steel A, et al. A cross-sectional examination of the profile of chiropractors recruited to the Australian Chiropractic Research Network (ACORN): a sustainable resource for future chiropractic research. BMJ Open. 2017;7(9): e015830.

17. Foley $\mathrm{H}$, Steel A. Patient perceptions of patient-centred care, empathy and empowerment in complementary medicine clinical practice: a cross-sectional study. Adv Integr Med. 2017;4(1): 22-30. 18. Bann CM, Sirois FM, Walsh EG. Provider support in complementary and alternative medicine: exploring the role of patient empowerment. J Altern Complement Med. 2010;16(7): 745752.

19. Glasgow RE, Wagner EH, Schaefer J, Mahoney LD, Reid RJ, Greene SM. Development and validation of the Patient Assessment of Chronic Illness Care (PACIC). Med Care. 2005;43(5): 436-444. 20. Foley $\mathrm{H}$, Steel A. Patient perceptions of clinical care in complementary medicine: a systematic review of the consultation experience. Patient Educ Couns. 2017;100(2): 212-223. 21. Sarsina PR di, Alivia M, Guadagni P. Traditional, complementary and alternative medical systems and their contribution to personalisation, prediction and prevention in medicine-personcentred medicine. EPMA Journal. 2012;3(1): 15. 
22. Hudon C, Fortin M, Haggerty J, Loignon C, Lambert M, Poitras M-E. Patient-centered care in chronic disease management: a thematic analysis of the literature in family medicine. Patient Educ Couns. 2012;88(2): 170-176.

23. Oberg EB, Thomas M-S, McCarty M, Berg J, Burlingham B, Bradley R. Older adults' perspectives on naturopathic medicine's impact on healthy aging. Explore. 2014;10(1): 34-43.

24. Rugg S, Paterson C, Britten N, Bridges J, Griffiths P. Traditional acupuncture for people with medically unexplained symptoms: a longitudinal qualitative study of patients' experiences. $B r J$ Gen Pract. 2011;61(587): e306.

25. Romeyke T, Nöhammer E, Scheuer HC, Stummer H. Integration of naturopathic medicine into acute inpatient care: an approach for patient-centred medicine under diagnosis-related groups. Complement Ther Clin Pract. 2017;28: 9-17.

26. Singer J, Adams J. Integrating complementary and alternative medicine into mainstream healthcare services: the perspectives of health service managers. BMC Complement Altern Med. 2014;14(1): 167.

27. Grace S, Higgs J. Integrative medicine: enhancing quality in primary health care. J Altern Complement Med. 2010;9.

28. Wardle J, Steel A, Casteleijn D, Bowman D. An evidence-based overview of naturopathic practice in Australia. Aust J Herbal Naturop Med. 2019;31(1): 9.

29. Stewart M. Towards a global definition of patient centred care. BMJ. 2001;322(7284): 444.

30. Mirzaei M, Aspin C, Essue B, et al. A patient-centred approach to health service delivery: improving health outcomes for people with chronic illness. BMC Health Serv Res. 2013;13(1): 251. 


\section{Figure Captions}

Figure 1. Details of interdependent measures.

Patient Centred Care Scale, Perceived Provider Support Scale, and Empowerment Scale items and scoring structure.

Figure 2. Details of Patient Assessment of Chronic Illness Care (PACIC).

PACIC domains, items and response option scoring structure. 